University of Warwick institutional repository: http://go.warwick.ac.uk/wrap This paper is made available online in accordance with publisher policies. Please scroll down to view the document itself. Please refer to the repository record for this item and our policy information available from the repository home page for further information.

To see the final version of this paper please visit the publisher's website. Access to the published version may require a subscription.

Author(s): S. Yim and T. S. Jones

Article Title: Growth dynamics of $\mathrm{C}_{60}$ thin films: Effect of molecular structure

Year of publication: 2009

Link to published version:

http://dx.doi.org/ 10.1063/1.3072805

Publisher statement: None 


\title{
Growth dynamics of $\mathrm{C}_{60}$ thin films: Effect of molecular structure
}

\author{
S. Yim ${ }^{1, a)}$ and T. S. Jones ${ }^{2}$ \\ ${ }^{1}$ Department of Chemistry, Kookmin University, Seoul 136-702, Republic of Korea \\ ${ }^{2}$ Department of Chemistry, University of Warwick, Coventry CV4 7AL, United Kingdom
}

(Received 6 November 2008; accepted 28 December 2008; published online 16 January 2009)

\begin{abstract}
The surface morphology and growth behavior of fullerene thin films have been studied by atomic force microscopy and height difference correlation function analysis. In contrast to the large growth exponents $(\beta)$ previously reported for other organic semiconductor thin-film materials, a relatively small $\beta$ value of $0.39 \pm 0.10$ was determined. Simulations of $(1+1)$-dimensional surface lateral diffusion models indicate that the evolution of deep grain boundaries leads to a rapid increase in $\beta$. We suggest that the commonly observed large $\beta$ values for organic thin films are due to their intrinsically anisotropic molecular structures and hence different stacking directions between crystallite domains. (C) 2009 American Institute of Physics. [DOI: 10.1063/1.3072805]
\end{abstract}

There is considerable interest in being able to control molecular thin-film growth and the resulting film morphology since molecular materials are finding increasing application in a wide range of optoelectronic devices, including organic light emitting diodes, photovoltaics, and field effect transistors. ${ }^{1}$ It is well known that device performance depends crucially on the structure and morphology of the organic films. ${ }^{2}$ Therefore, detailed knowledge of the film growth mechanisms and the resulting morphological properties is essential both from fundamental and technological perspectives. Nonetheless, current understanding of the growth behavior of molecular thin films is limited, in particular when compared to their well-studied inorganic semiconductor and metal thin-film counterparts. Molecular materials offer several unique features, which complicate the growth process. For example, they do not possess spherical symmetry and hence diffusion depends generally on the orientation of the neighboring molecules or crystallites. Consequently, the small number of studies on the growth behavior of molecular films reports very different characteristics from those commonly observed for inorganic and metallic thin films. $^{3-7}$

One of the most important differences is the large value of the growth exponent $(\beta)$ obtained through application of scaling analysis. For example, recent studies of free-base phthalocyanine $\left(\mathrm{H}_{2} \mathrm{Pc}\right){ }^{3}$ di-indenoperylene, ${ }^{5}$ and perylene$3,4,9,10$-tetracarboxylic dianhydride (PTCDA) ${ }^{7}$ report $\beta$ values of $1.02,0.75$, and 0.54 , respectively. These values are significantly larger than those obtained for inorganic thin films ${ }^{8-11}$ and greater than that predicted for the random deposition model, where $\beta_{\mathrm{RD}}=0.5$. $^{12}$ Random deposition is expected to define an upper bound on the growth of the roughness unless the depositing particles are allowed to transport to higher layers, e.g., in the case of dewetting. ${ }^{12,13}$ In this context, systems with $\beta>\beta_{\mathrm{RD}}=0.5$ imply an unusually rapid roughening. It has been suggested that this rapid roughening in molecular thin-film growth can be explained by spatial inhomogeneities due to the presence of different tilt domains and grain boundaries, ${ }^{3-5}$ a consequence of the structural anisotropy of the molecular materials studied.

\footnotetext{
${ }^{a)}$ Electronic mail: sgyim@kookmin.ac.kr.
}

In this letter, we present results for the growth of fullerene $\left(\mathrm{C}_{60}\right)$ thin films, a molecule widely used in many organic optoelectronic devices. Measurements of the dynamic scaling exponents for this relatively isotropic molecule reveal an unusually low $\beta$ value. Analysis of a simple lateral diffusion growth model, which includes the presence of grain boundaries, provides further insight into the role of molecular structure and anisotropy in determining the morphological properties of the growing film.

The $\mathrm{C}_{60}$ films were grown in an organic molecular beam deposition chamber with a base pressure of $\sim 2 \times 10^{-9}$ Torr. Commercially available $\mathrm{C}_{60}$ (MER Corp., 99.5\%) molecules were outgassed for $20 \mathrm{~h}$ before growth and sublimed onto quartz substrates held at room temperature using a miniature effusion cell. The cell temperature was $\sim 400{ }^{\circ} \mathrm{C}$, which corresponds to a growth rate of $\sim 1.3 \AA \mathrm{s}^{-1}$ as determined by a quartz crystal microbalance positioned near the substrate and UV absorption spectroscopy. Ex situ surface morphologies at different film thicknesses were observed using tapping mode atomic force microscopy (AFM).

Representative AFM images of $\mathrm{C}_{60}$ thin films of different thicknesses $(D=500-8000 \AA)$ are shown in Fig. 1. All images were taken with the same scan area of 1.0 $\times 1.0 \mu \mathrm{m}^{2}$. Morphological analysis was carried out using height difference correlation function (HDCF) analysis, where the growth and roughness scaling follow simple laws characterized by roughness $(\alpha)$, growth $(\beta)$, and dynamic $(z)$ scaling exponents, which can be determined from the meansquare fluctuation $g(R)$,

$$
\begin{aligned}
& g(R)=\left\langle\left[h(x, y)-h\left(x^{\prime}, y^{\prime}\right)\right]^{2}\right\rangle, \\
& R=\sqrt{\left(x-x^{\prime}\right)^{2}+\left(y-y^{\prime}\right)^{2}} .
\end{aligned}
$$

The average is taken over all pairs of points $(x, y)$ and $\left(x^{\prime}, y^{\prime}\right)$ separated laterally by the length, $R$. There are two distinct regimes which depend on the relative magnitudes of $R$ and the correlation length, $\xi ; g(R) \propto R^{2 \alpha}$ for $R \ll \xi$ and $g(R)=2 \sigma^{2}$ for $R \gg \xi$, where $\sigma$ is the mean-square surface roughness. The parameters $\sigma$ and $\xi$ are related to the film thickness, $D$, according to the power laws $\sigma \propto D^{\beta}$ and $\xi$ $\propto D^{1 / z}$.,14 The correlation length, $\xi$, at each thickness was determined by fitting the HDCF to the following analytical function: $g(R)=2 \sigma^{2} \widetilde{g}(R / \xi)$, where $\widetilde{g}(x)=1-\exp \left(-x^{2 \alpha_{\text {loc }}}\right) .{ }^{15,16}$ 

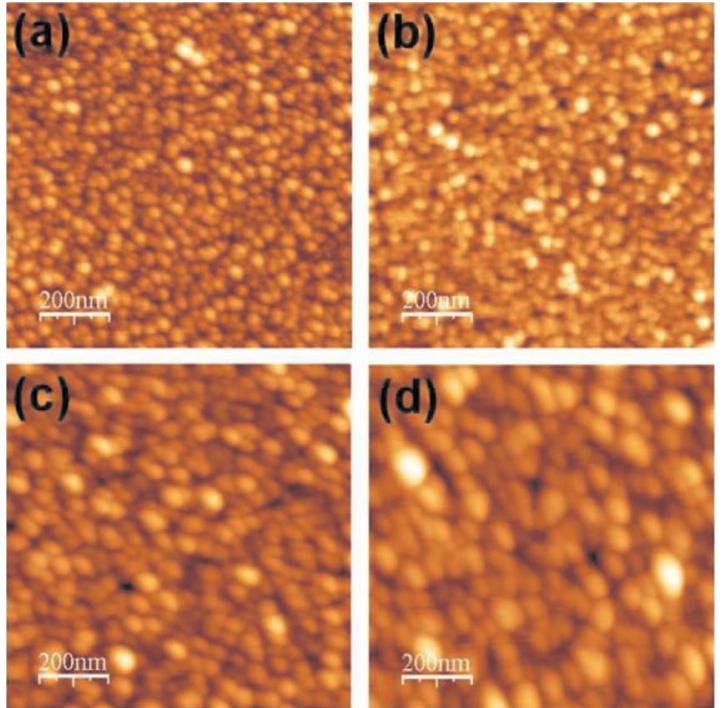

FIG. 1. (Color online) Room temperature AFM images of $\mathrm{C}_{60}$ thin films deposited on quartz substrates with various thicknesses, $D$, of (a) $500 \AA$, (b) $1000 \AA$, (c) $4000 \AA$, and (d) $8000 \AA$.

It has been shown in many cases that $\alpha, \beta$, and $z$ are not independent and obey conventional scaling, i.e., $1 / z$ $\cong \beta / \alpha$. ${ }^{5,6,14}$

Figure 2(a) shows a plot of the mean-square surface fluctuation, $g(R)$, as a function of lateral distance, $R$, for different values of $D$. By fitting $g(R) \propto R^{2 \alpha_{\text {loc }}}$ in the range of $R \ll \xi$, an average value of $\alpha_{\text {loc }}=0.77 \pm 0.15$ is determined [Fig. 2(b)]. The surface roughness values $(\sigma)$ are plotted in Fig. 2(c) as a function of $D$ and this yields a $\beta$ value of $0.39 \pm 0.10$ from the power law, $\sigma \propto D^{\beta}$. Figure 2(d) shows a plot of $\xi$ as a function of $D$ and yields $1 / z=0.34 \pm 0.12$. The value obtained for $\beta / \alpha_{\text {loc }}(=0.51 \pm 0.2)$ is $\sim 1.5$ times larger than the $1 / z$ value. This discrepancy between $\beta / \alpha_{\text {loc }}$ and $1 / z$ has been reported for several molecular thin-film systems. For example, $\beta / \alpha_{\text {loc }}$ is $\sim 2.4$ times and $\sim 3.0$ times larger
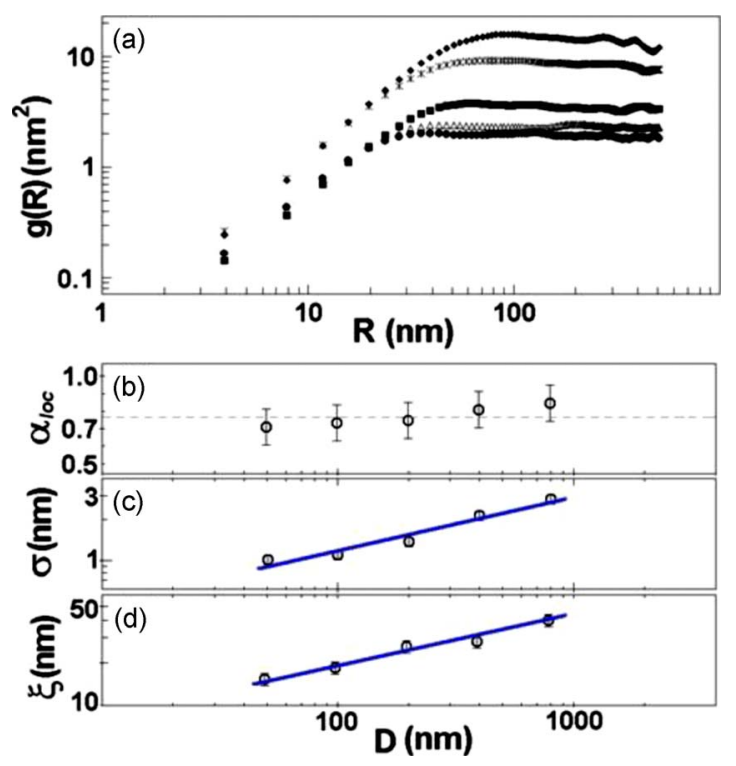

FIG. 2. (Color online) (a) Plot of averaged $g(R)$ vs $R$ for $\mathrm{C}_{60}$ films with various $D(\boldsymbol{O}=500 \AA, \triangle=1000 \AA, \quad=2000 \AA, x=4000 \AA$, and $\bullet$ $=8000 \AA$ A). Also shown are plots of (b) $\alpha_{\text {loc }}$, (c) $\sigma$, and (d) $\xi$ as a function of film thickness, $D$. The horizontal dashed line in (b) denotes the average value of $\alpha_{\text {loc }}$.
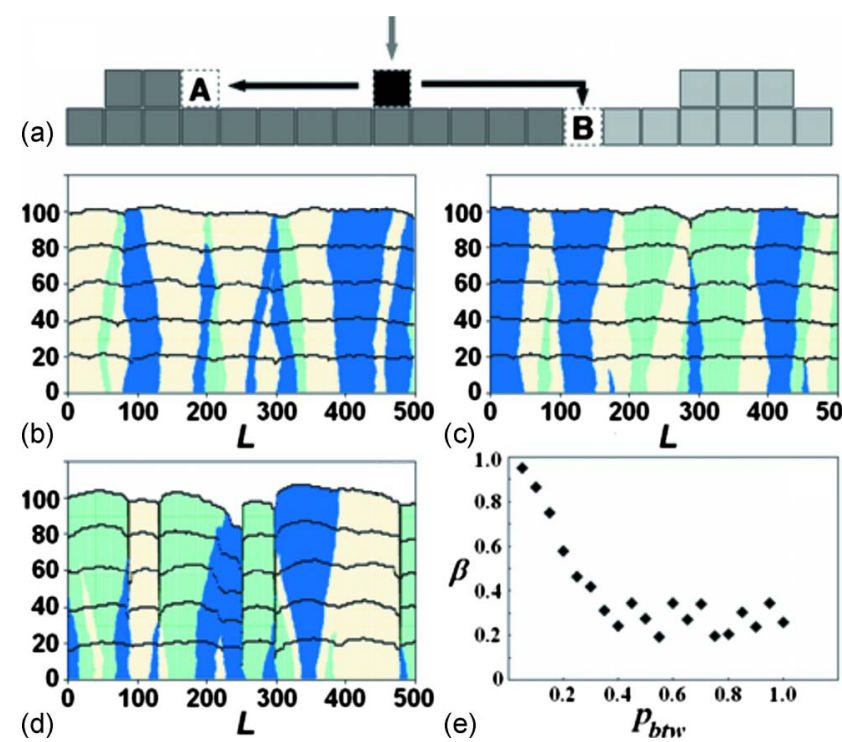

FIG. 3. (Color online) (a) Simple $(1+1)$-dimensional lateral diffusion model. Representative surface growth patterns generated by the model with various $p_{\text {btw }}$ values of (b) 0.5 , (c) 0.3 , and (d) 0.1 . The total number of columns is 500 and thick curves are drawn after the deposition of each set of 10000 particles. (e) Plot of growth exponents, $\beta$, as a function of $p_{\mathrm{btw}}$.

than the $1 / z$ values for $\mathrm{H}_{2} \mathrm{Pc}$ and PTCDA thin films, respectively. It has been suggested that this discrepancy arises from the existence of a high potential energy barrier at the step edges of existing islands. ${ }^{3,7}$ It is most pronounced for PTCDA, a planar molecule which grows with its molecular planes parallel to the substrate to maximize the intermolecular interactions in a direction normal to the surface. This configuration leads to a very high step edge barrier and the large discrepancy between $\beta / \alpha_{\text {loc }}$ and $1 / z$ can be rationalized by the breakage of the parallel configuration at the step edges when the molecule moves down to the lower layer. ${ }^{7}$ By contrast, the spherical shape of the $\mathrm{C}_{60}$ molecules should lead to a relatively small deviation from conventional scaling, although the results suggest that a small step edge barrier is present.

A decrease in the crystallographic ordering of the films may also lead to reduced step edge barriers and indeed several studies have reported significant differences in the $\beta$ values obtained for amorphous and crystalline films. ${ }^{17,18}$ $\mathrm{C}_{60}$ thin films grow epitaxially on well-defined metallic substrates, ${ }^{19,20}$ but island growth occurs on relatively weak interacting substrates. ${ }^{21}$ For growth on quartz substrates such a weak substrate-molecule interaction would reduce the crystallographic ordering of the $\mathrm{C}_{60}$ molecules and hence lead to a reduced step edge barrier and a low $\beta$ value.

The large $\beta$ values obtained for most molecular thin-film material systems ${ }^{3,5,7}$ are most likely a consequence of large step edge barriers and deep grain boundaries between neighboring domains with different molecular orientations, which comes from the intrinsic anisotropy of the molecular structures and their crystallographic ordering. To investigate in greater detail the influence of deep grain boundaries on the growth exponents, simple $(1+1)$-dimensional surface growth models have been simulated and are presented in Fig. 3. Organic molecules are bonded to each other by relatively weak van der Waals forces, which cause the impinging molecules to diffuse laterally across the surface [Fig. 3(a)]. In these simulations, deposited particles are allowed to diffuse 
along the surface up to a finite distance (ten columns maximum) before stopping when they find an energetically stable position. For each simulation, 50000 particles were randomly deposited on a substrate consisting of 500 horizontal columns, $L=500$. The occurrence of the grain boundaries and accompanying rapid increase in roughness are simulated by varying $p_{\text {btw }}$, which is defined as the probability that the position between the grains with different orientations, e.g., position B in Fig. 3(a), accommodates the particle. For example, if $p_{\mathrm{btw}}=1$, the particle can occupy the position without any restriction; however when $p_{\mathrm{btw}}=0$, the particle is not allowed to fall into the position at all. Several examples of simulation results are represented in Figs. 3(b)-3(d) with various $p_{\text {btw }}$ values of $0.5,0.3$, and 0.1 , respectively. Thick curves in the figures are drawn after the deposition of each set of 10000 particles so that the increase in roughness and the evolution of grain boundaries can be easily recognized. It is also assumed that the particles are allowed to have three different stacking directions and no step edge barrier exists for all cases. In the figures, different shading denotes different orientation. The initially deposited particles can randomly adopt one of the three orientations and subsequent particles sticking to existing grains by lateral diffusion have the same stacking direction as the initial grains. Deep grain boundaries in organic crystalline thin films occur when the molecules cannot occupy the position between the two grains with different stacking directions since the intermolecular interactions between the deposited molecule and existing molecules in both sides cannot be optimized simultaneously. The obtained $\beta$ values in these simulations show a strong tendency to increase with the decrease in $p_{\mathrm{btw}}$ when $p_{\mathrm{btw}}$ is $<0.4$ [Fig. 3(e)]. For $p_{\mathrm{btw}}>0.4, \beta$ values of $0.20-0.35$ are obtained, which are consistent with those observed in many conventional atomic thin-film systems. In the range of $p_{\text {btw }}$ $<0.4$, however, $\beta$ increases very rapidly and approaches unity as $p_{\text {btw }}$ becomes very small. When $p_{\text {btw }}=0.20$, the $\beta$ value is 0.58 and larger than $\beta_{\mathrm{RD}}(=0.5)$. In organic crystalline thin films deposited on weakly interacting substrates such as glass, the stacking direction of each crystalline domain is not determined uniquely and hence the formation of deep grain boundaries is inevitable. The simulations suggest that the commonly observed large $\beta$ values for organic thin films can be rationalized by these deep boundaries due to the intrinsic anisotropy of molecules, which results in different stacking directions between crystallite domains.
In conclusion, the growth behavior of $\mathrm{C}_{60}$ thin films on quartz substrates has been studied using AFM and HDCF analysis. $\beta$ values of $0.39 \pm 0.10$ are obtained, which are much smaller than those reported previously for other molecular thin films. The experimental results and simulations of a simple $(1+1)$-dimensional surface growth model, which includes deep grain boundaries, suggest that the commonly observed large $\beta$ values for organic thin films arise from the intrinsic anisotropy of molecules, which leads to different stacking directions between crystallite domains.

This work was supported by the Korea Research Foundation grant funded by the Korean Government (MOEHR, Basic Research Promotion Fund) (Grant No. KRF-2008-331C00138) and Research Program 2007 of Kookmin University in Korea.

${ }^{1}$ S. R. Forrest, Chem. Rev. (Washington, D.C.) 97, 1793 (1997).

${ }^{2}$ D. J. Gundlach, Y. Y. Lin, T. N. Jackson, S. F. Nelson, and D. G. Schlom, IEEE Electron Device Lett. 18, 87 (1997).

${ }^{3}$ S. Yim and T. S. Jones, Phys. Rev. B 73, 161305 (2006).

${ }^{4}$ S. Kowarik, A. Gerlach, and F. Schreiber, J. Phys.: Condens. Matter 20, 184005 (2008).

${ }^{5}$ A. C. Dürr, F. Schreiber, K. A. Ritley, V. Kruppa, J. Krug, H. Dosch, and B. Struth, Phys. Rev. Lett. 90, 016104 (2003).

${ }^{6}$ G. W. Collins, S. A. Letts, E. M. Fearon, R. L. McEachern, and T. P. Bernat, Phys. Rev. Lett. 73, 708 (1994).

${ }^{7}$ S. Yim K.-I Kim, and T. S. Jones, J. Phys. Chem. C 111, 10993 (2007).

${ }^{8}$ J. Krug, Adv. Phys. 46, 139 (1997).

${ }^{9}$ J. H. Jeffries, J.-K. Zuo, and M. M. Craig, Phys. Rev. Lett. 76, 4931 (1996).

${ }^{10}$ S. Huo and W. Schwarzacher, Phys. Rev. Lett. 86, 256 (2001).

${ }^{11}$ J. M. Lopez, M. Castro, and R. Gallego, Phys. Rev. Lett. 94, 166103 (2005).

${ }^{12}$ A. L. Barabasi and H. E. Stanley, Fractal Concepts in Surface Growth (Cambridge University Press, Cambridge, 1995).

${ }^{13}$ J. Krug, Physica A 340, 647 (2004).

${ }^{14}$ Y.-P. Zhao, J. B. Fortin, G. Bonvallet, G.-C. Wang, and T.-M. Lu, Phys. Rev. Lett. 85, 3229 (2000).

${ }^{15}$ G. Palasantzas and J. Krim, Phys. Rev. B 48, 2873 (1993).

${ }^{16}$ V. Holý, U. Pietsch, and T. Baumbach, High-Resolution X-Ray Scattering From Thin Films and Multilayers (Springer, Berlin, 1999).

${ }^{17}$ S. Kowarik, A. Gerlach, S. Sellner, F. Schreiber, J. Pflaum, L. Cavalcanti, and O. Konovalov, Phys. Chem. Chem. Phys. 8, 1834 (2006).

${ }^{18}$ T.-W. Huang, H.-Y. Lee, Y.-W. Hsieh, and C.-H. Lee, J. Cryst. Growth 237, 492 (2002).

${ }^{19}$ J. T. Sadowski, R. Z. Bakhtizin, A. I. Oreshkin, T. Nishihara, A. AlMahboob, Y. Fujikawa, K. Nakajima, and T. Sakurai, Surf. Sci. Lett. 601, L136 (2007).

${ }^{20}$ W. W. Pai and C-L. Hsu, Phys. Rev. B 68, 121403 (2003).

${ }^{21}$ P. Reinke, H. Feldermann, and P. Oelhafen, J. Chem. Phys. 119, 12547 (2003). 